\title{
Replantation of an Avulsed Primary Incisor: Report of a Case with Favorable Outcome
}

\author{
${ }^{1}$ Aditi Kapur, ${ }^{2}$ Ashima Goyal, ${ }^{3}$ Krishan Gauba
}

\begin{abstract}
Replantation of an avulsed primary tooth is not recognized as a treatment option due to the possibility of several unfavorable outcomes and insufficient documented clinical evidence to bring about any change in the protocols. There are a few case reports, however, which have shown successful results. The following paper reports a successfully carried out replantation procedure in a 3-year and 2-month-old female child and discusses possible reasons for a favorable outcome.
\end{abstract}

Keywords: Avulsion, Primary tooth, Replantation.

How to cite this article: Kapur A, Goyal A, Gauba K. Replantation of an Avulsed Primary Incisor: Report of a Case with Favorable Outcome. J Postgrad Med Edu Res 2014;48(2):105-108.

Source of support: Nil

Conflict of interest: None

\section{INTRODUCTION}

Tooth avulsion is the most severe form of tooth displacement due to trauma and is defined as complete loss of a tooth out of its bony socket. ${ }^{1}$ Primary teeth tend to become avulsed or displaced more often, rather than fracture, because of a resilient alveolar bone, which cushions and dissipates the impact of trauma. ${ }^{2}$ The prevalence of primary tooth avulsions has been reported to be nearly $0.8 \%{ }^{3}$ and it comprises around 7 to $14 \%$ of all injuries to the primary dentition. ${ }^{3,4}$ Maxillary incisors are the most commonly affected teeth and the most commonly avulsed primary tooth is the right central incisor. ${ }^{3,4}$

Replantation of an avulsed primary tooth is not yet accepted as a treatment option in the guidelines formulated by either American Academy of Pediatric Dentistry ${ }^{5}$ or International Association of Dental Traumatology ${ }^{6}$ with a rationale that treatment selections should be aimed at minimizing any additional risks of further damage to the permanent successors, keeping in view the close proximity of the underlying permanent tooth germ, to the apex of roots of the injured primary tooth.

\footnotetext{
${ }^{1}$ Assistant Professor, ${ }^{2,3}$ Professor

${ }^{1-3}$ Oral Health Sciences Centre, Postgraduate Institute of Medical Education and Research, Chandigarh, India

Corresponding Author: Aditi Kapur, Assistant Professor Oral Health Sciences Centre, Postgraduate Institute of Medical Education and Research, Chandigarh, India, Phone: 9815966348, e-mail: draditikmalhotra@mail.com
}

Replantation as a treatment choice for an avulsed primary incisor, has been therefore, viewed with much caution, and not been widely attempted and remains debatable, in spite of a few successful case reports. ${ }^{7-12}$

The maximum amounts of avulsion injuries have been reported to occur between 2 and 4 years of age. ${ }^{3,4}$ This is the time when the child is learning to become more independent and is also developing self-esteem by interaction with his peers and adults. These days children are extremely conscious of their appearance and also sometimes guide treatment toward more esthetic options. A loss of a front tooth at such a young impressionable age can, therefore, have a profound effect on the psychological and social well being of the child. Pediatric dentists often see a boost in the child's self confidence after rehabilitation of front teeth lost due to caries. It may, therefore, be worthwhile to attempt to preserve a traumatically lost anterior tooth in a young, growing child.

The following paper reports a successful case of replantation of a primary anterior avulsed tooth in a 3-year 2-month-old female child, followed uneventfully, till complete eruption of the permanent successor and also discusses various factors responsible for a favorable outcome.

\section{CASE REPORT}

A 3-year 2-month-old female child was brought to the emergency care of Unit of Pediatric Dentistry by both her parents. She was the only child to her parents, who were physicians at the Postgraduate Institute of Medical Education and Research, Chandigarh. The child was awake and oriented and had suffered a traumatic dental injury due to slipping and hitting the basin leading to avulsion of a primary central incisor which was brought placed in milk by the parents. An extraoral examination revealed mild bruises on the left lip. Intraorally, an empty socket of 61 along with a subluxated 51 with no associated dentoalveolar fracture or injury was found. There was no tooth affected with dental caries. History from the parents revealed an extraoral dry time of nearly 15 minutes, followed by immediate placement of the tooth in milk for nearly an hour till they reached the Pediatric dental emergency services. The avulsed tooth had a fully formed and intact root, which was rinsed with saline and placed in Hank's balanced salt solution, ${ }^{5}$ a tissue culture medium, 
available in the department, for 30 minutes. Two treatment options were considered and explained to the parents along with the rationale and limitations: (i) immediate extraction, followed by prosthetic rehabilitation later, (ii) replantation and splinting, followed by root canal treatment. The parents gave consent for the latter procedure, primarily to avoid the immediate negative psychological impact of loss of a front tooth to the child as well as themselves. The child had no previous dental history, was distressed due to the episode of trauma, but complied well during dental examination. Local anesthesia was administered and 61 was manipulated back into the socket with ease. A Multiflex wire and composite splint was placed from 53 to 63 for stabilization. A behavior modification using combination of TSD, TLC, distraction and contingency management were used to maintain the child's co-operation throughout the procedure, which lasted for about 30 minutes. The parents were given oral hygiene instructions and advised to give a soft diet to the child till removal of the splint. At a follow-up examination a day later the child was found to be comfortable and symptom-free. An intraoral periapical X-ray was taken which showed a well-aligned root of 61 (Fig. 1). Root canal treatment was initiated and access opening and pulpectomy was done. The parents, however, could not report for completion of RCT on account of being away and got the treatment completed from a local private practitioner. They reported after 2 months with an asymptomatic 61 showing no discoloration or abnormal mobility. The splint had been removed. An intraoral periapical radiograph was taken which revealed a well-obturated root canal of 61 (Fig. 2). A follow-up exami-nation 8 months after replantation showed a slightly mobile 61. An IOPA revealed beginning of external root resorption in relation to root of 61 (Fig. 3). Two months later, however, the mobility increased and was scored as grade 2 . A decision to remove the root canal filling material was,

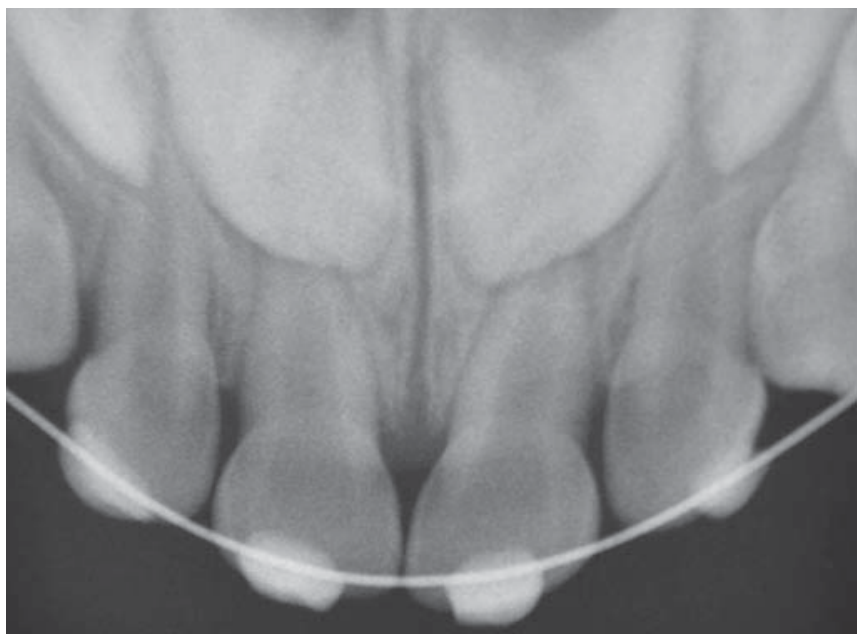

Fig. 1: IOPA taken after replantation of avulsed 61 and splinting therefore, taken, which led to reduction in mobility. An IOPA at this stage revealed no significant increase in resorption (Fig. 4). The canal was thoroughly irrigated with saline and sealed with composite. The patient was now reviewed after every 2 months, and found to be asymptomatic, apart from slight mobility of 61. Eighteen months after replantation, however, there was an increase in mobility and an IOPA showed resorption of more than half the root of 61 (Fig. 5). The mobility, however, did not cause any discomfort to the patient. A treatment plan of extraction of 61 and splinting of crown of 61 with the adjacent teeth was considered at this stage, in case of further increase in mobility. The patient, however, remained asymptomatic and an IOPA taken 32 months after replantation (Fig. 6) showed a completely resorbed root of 61 . Four months later 61 exfoliated and the underlying permanent incisor erupted uneventfully (Fig. 7).

\section{DISCUSSION}

Goal of any treatment rendered in the primary dentition is to preserve the integrity of the underlying permanent dentition.

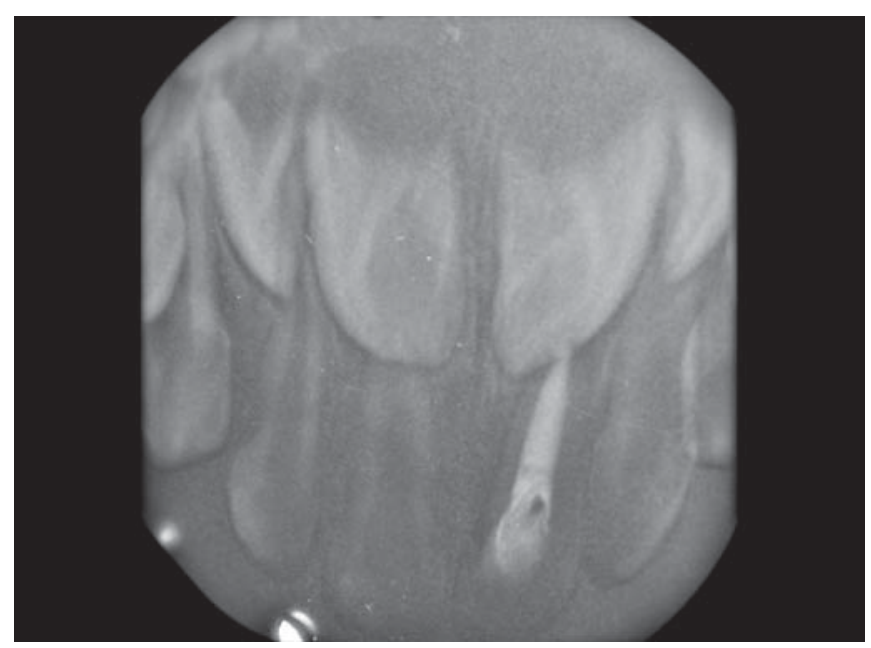

Fig. 2: IOPA taken after root canal treatment in 61, 2 months after replantation

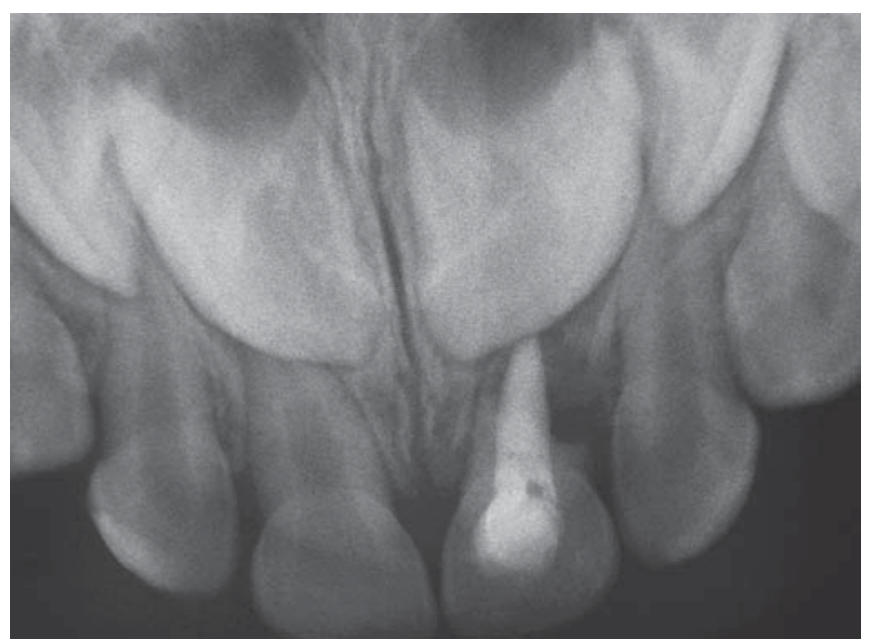

Fig. 3: IOPA taken 8 months after replantation showing external resorption in root of 61 
It is important to remember, that there exists a close relationship between the apex of the primary tooth and the germ of the permanent successor. ${ }^{6}$ Therefore, primary tooth avulsion as a traumatic dental injury has been shown to result in developmental disturbances such as tooth malformation, crown discolorations, and hypoplasia in the permanent dentition nearly 30 to $60 \%$ of times. ${ }^{3,4}$ Additionally, the pathological sequelae associated with the attempted replantation, such as inadvertent damage to the underlying permanent tooth germ while manipulating the crown back in the socket, development of a dental abscess, ankylosis may also affect the development and eruption of the underlying tooth. ${ }^{13}$

In the present report, however, none of these consequences were seen and despite showing external resorption, which was slowly progressing, the replanted 61 was able to maintain the integrity of the arch and esthetics for the child till the eruption of the permanent successor. The underlying permanent successor, i.e. 21 erupted in tandem with the contralateral and did not have any morphological defects (see Fig. 7). Care had been taken to gently manipulate the 61

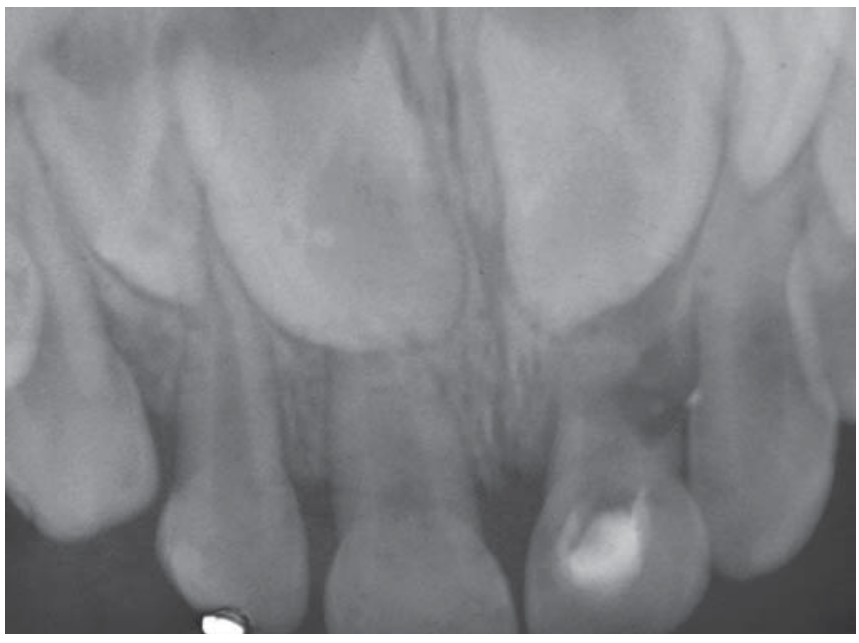

Fig. 4: IOPA taken after 10 months after replantation showing persistent resorption of 61 root

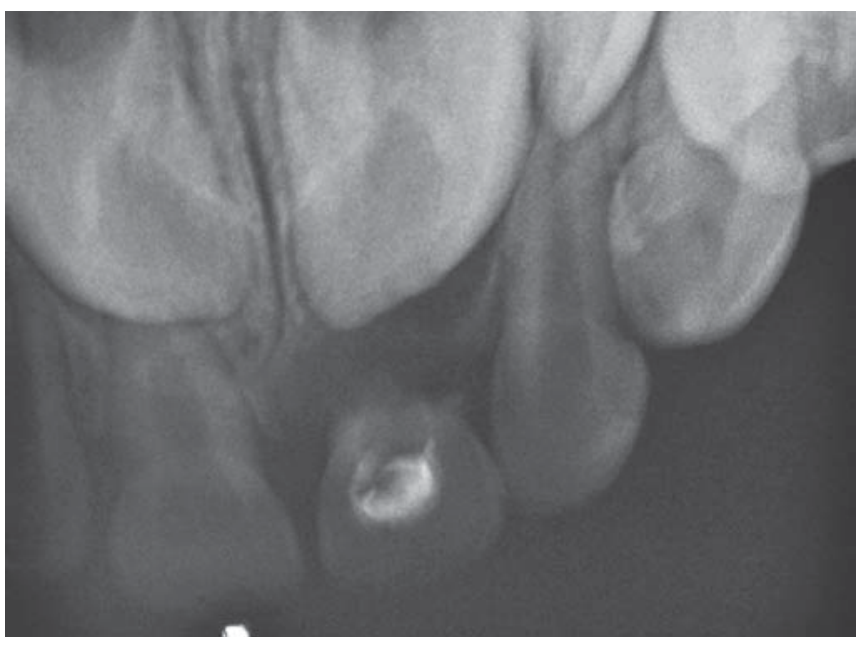

Fig. 5: IOPA taken after 18 months after replantation showing near complete resorption of root of 61 back in the socket. Some authors have recommended resecting the root tip which is about a quarter or one-fifth of the total length of root of the tooth, retrograde endodontic preparation and filling prior to replanting an avulsed primary tooth in order to prevent any damage to the underlying dental germ. ${ }^{14}$

There are many other clinicians who have reported successful outcomes with this procedure. Kawashima and Pineda $^{7}$ (1992) reported replantation without any splint of two avulsed mandibular incisors lying attached with a little gingival tissue intraorally in a 2-year-old girl, which remained aymptomatic, and without any crown discoloration, abnormal mobility and without pain or discomfort to the patient at a 2-year follow-up. Weiger and Heuchert ${ }^{8}$ (1999) reported a case of an avulsed upper left primary incisor in a 3 and a half-year-old girl child where the tooth was still in contact with the vestibular mucosa and was managed by replantation, splinting with a metal wire and root canal treatment after 11 days. The tooth developed a fistula after 18 months and had to be extracted due to progressive external resorption. Kinoshita et $\mathrm{al}^{9}$ (2000) reported replantations of eight avulsed incisors in six patients aged 9 months to 3 years 9 months. Resin splints were used for stabilization, except in one case where suture had been placed. Four teeth remained asymptomatic and four had to be extracted due to extreme mobility. The authors concluded that replantation could be an effective treatment when the condition of the avulsed primary tooth is found suitable. The permanent counterpart, however, erupted 6 months later without any pathological findings. Kubo et $\mathrm{al}^{10}$ (2005) reported a successful case of replantation in a 2-year one month-old boy of an avulsed 51 which was brought placed in milk nearly an hour and a half after trauma. Following replantation and splinting with orthodontic ligature wires and composite, endodontic treatment was carried out after 25 days due to crown discoloration. The tooth showed progressive root resorption and remained in

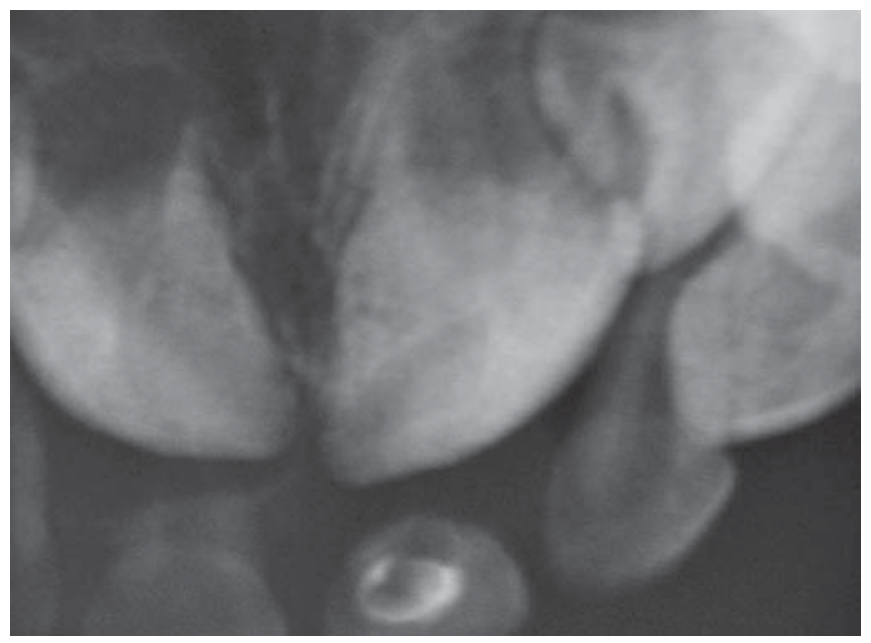

Fig. 6: IOPA taken after 32 months after replantation showing 61 crown to be still in place 


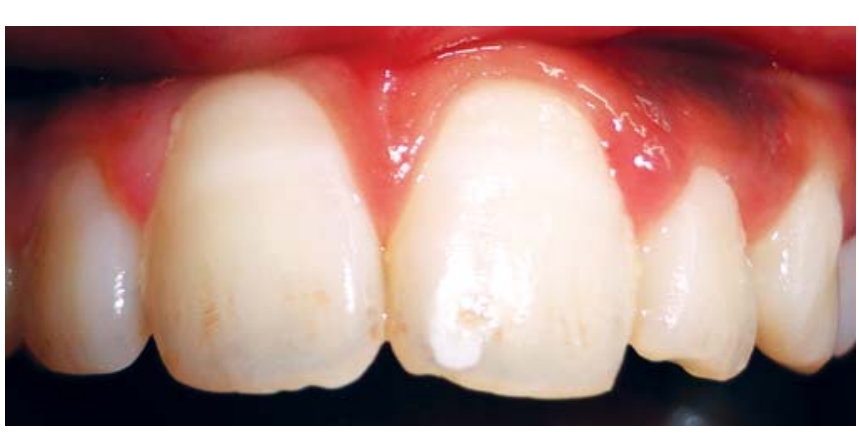

Fig. 7: Erupted permanent incisors

place till normal eruption of the succedaneous tooth. Rocha and Cardoso ${ }^{11}$ (2006) reported a successful replantation, splinting with a 0.5 steel wire, of an avulsed 61 in a 2-year 6-month-old child, with an extra-alveolar time of 30 minutes, stored in milk. The tooth was endodontically treated after a few days and was followed up till eruption of the permanent incisor, which had no esthetic alterations. Friedlander et al ${ }^{12}$ (2012) reported a case of replantation and stabilization of 61 with sutures in a 9-month-old boy, traumatically avulsed during a tonsillectomy surgery, which exfoliated naturally after 6 years and the 21 erupted uneventfully. In the absence of any guidelines, the reported cases too, lack consistency in terms of the management techniques employed. The current evidence for replantation of primary teeth is, therefore, experimental, descriptive and base on individual opinions (level III). ${ }^{13}$

Zamon et $\mathrm{al}^{13}$ summarized various factors as risks vs benefits for replanting primary teeth. The risks outlined were committing a child to additional dental treatment involving local anesthesia, radiographs, splinting and endodontics and possibility of pathological outcomes mentioned earlier. The possible benefits include space maintenance, prevention of abnormal habits like tongue thrust, and negatively affecting child's self-esteem. The positive factors responsible for a successful outcome in the present case were an extraoral dry time of less than 15 minutes, following which the avulsed tooth was placed in milk and HBSS; a compliant patient and parental desire for restoration of esthetics and their easy availability for follow-up visits.

\section{CONCLUSION}

The present scientific evidence may fully justify the guidelines of not replanting a primary tooth. It may, however, be attempted in specific cases where the clincian is able to identify a minimum risk vs benefit ratio.It cannot, however, be considered as a definitive treament and a frequent regular follow-up is mandatory for monitoring any pathologic sequelae. Documentation of a tempted procedures with associated variables influencing a successful or unsuccessful clinical outcomes is important, to generate more evidence either in favor or against this treatment modality.

\section{REFERENCES}

1. Andreason JO, Andreasen FM, Andreasen L. Textbook and colour atlas of traumatic injuries to the teeth. 4th ed. Blackwell Munksgaard Publishers 2007.

2. Kupietzky A. The treatment and long-term management of severe multiple avulsions of primary teeth in a 19-month-old child. Pediatr Dent 2001;23(6):517-521.

3. Christophersen P, Freund M, Harild L. Avulsion of primary teeth and sequalae on the permanent successors. Dental Traumatology 2005;21:320-323.

4. Lenzi MM, Jacomo DR, Carvalho V, Campos V. Avulsion of primary teeth and sequelae on the permanent successors: longitudinal study. Brazilian J Dent Traumatology 2011;2(2):80-84.

5. Council O. Guidelines on management of acute dental trauma. Reference Manual 2011;34(6):230-238.

6. Malmgren B, Andreasen JO, Flores MT, Robertson A, DiAngelis AJ, Andersson L, Cavalleri G, Cohenca N, Day P, Hicks ML, et al. International Association of Dental Traumatology guidelines for the management of traumatic dental injuries: 3. Injuries in the primary dentition. Dental Traumatology 2012;28:174-182.

7. Kawashima Z, Pineda LF. Replanting avulsed primary teeth. JADA 1992;123:90-92.

8. Weiger R, Heuchert T. Management of an avulsed primery incisor. Endod Dent Traumatol 1999;15:138-143.

9. Kinoshita S, Mitomi T, Taguchi Y, Noda T. Prognosis of replanted primary incisors after injuries. Endod Dent Traumatol 2000;16: 175-183.

10. Kubo S, Kadoya M, Takeuchi T, Yakusiji M. Replantation of an avulsed primary central incisor. J Hard Tissue Biology 2005; 14(2):154-156.

11. Rocha MJC, Cardoso M. Reimplantation of primary tooth—case report. Dental Traumatology 2008;24:e4-e10.

12. Friedlander LT, Chandler NP, Drummond BK. Avulsion and replantation of a primary incisor tooth. Dental Traumatolo 2012. doi: 10.1111/j.1600-9657;1-4.

13. Zamon EL, Kenny DJ. Replantation of avulsed primary incisors: a risk- benefit assessment. J Can Dent Assoc 2001;67:386-391.

14. Fillipi A, Pohl Y, Kirschner H. Replantation of avulsed primary anterior teeth: treatment and limitations. ASDC 1997;64(4): 272-275. 